\title{
Differences in the Nonverbal Requests of Great Apes and Human Infants
}

\author{
Marloes H. van der Goot \\ Max-Planck-Institute for Psycholinguistics
}

\author{
Michael Tomasello \\ Max-Planck-Institute for Evolutionary Anthropology
}

\author{
Ulf Liszkowski \\ Max-Planck-Institute for Psycholinguistics and University of Hamburg
}

\begin{abstract}
This study investigated how great apes and human infants use imperative pointing to request objects. In a series of three experiments (infants, $N=44$; apes, $N=12$ ), subjects were given the opportunity to either point to a desired object from a distance or else to approach closer and request it proximally. The apes always approached close to the object, signaling their request through instrumental actions. In contrast, the infants quite often stayed at a distance, directing the experimenters' attention to the desired object through indexfinger pointing, even when the object was in the open and they could obtain it by themselves. Findings distinguish 12-month-olds' imperative pointing from ontogenetic and phylogenetic earlier forms of ritualized reaching.
\end{abstract}

It is widely believed that human imperative pointing to request objects is somehow simpler than human declarative pointing to share attention (e.g., Camaioni, 1993; Gómez, Sarriá, \& Tamarit, 1993; Tomasello \& Camaioni, 1997). One piece of evidence for this proposal is that, in some studies at least, imperative pointing emerges first in human ontogeny (Camaioni, Perucchini, Bellagamba, \& Colonnesi, 2004; but see Carpenter, Nagell, \& Tomasello, 1998). Moreover, infants' earliest imperative gestures are often effected by more open-hand pointing rather than index-finger pointing, resembling a kind of ritualized reaching (Blake et al., 1994). Another piece of evidence is that children with autism point imperatively but not declaratively (Baron-Cohen, 1989). Moreover, although nonhuman primates do not point at all for one another in their natural communication, captive great apes do not point declaratively but do point for humans imperatively to request objects (Call \& Tomasello, 1994; Leavens \& Hopkins, 1999).

We thank Mireille Hassemer, Birgit Knudsen, Rocio Silva Zunino, Stefanie Voigt, and Johanna van Schaik for help with the data collection, and Loraen Kaltenschnee, Mireille Hassemer, and Lena Schumacher for help with the coding. Special thanks go to Johannes Grossmann from the Max-Planck-Institute for Evolutionary Anthropology for his essential support and assistance in data collection.

Correspondence concerning this article should be addressed to Ulf Liszkowski, Department of Developmental Psychology, University of Hamburg, Von-Melle-Park 5, 20146 Hamburg, Germany. Electronic mail may be sent to ulf.liszkowski@ uni-hamburg.de.
This traditional analysis relies on a characterization of imperative pointing as ritualized reaching and declarative pointing as the sharing of mental states. However, there are at least two interpretations of imperative object requests (Bruner, 1975). On a lean level, gestural requests do indeed reflect the signaling of an individual action attempt to obtain an object. Such signaling originates from ontogenetically ritualized instrumental behaviors, like reaching. The intention becomes apparent in the instrumental action pattern ("instrumental request"). In contrast, on a richer level requests are part of human cooperative communication. They work by directing another's attention to something within common ground, with the expectation that attending to that thing will lead her to infer the communicator's communicative intention. The gesture is "decontextualized" from instrumental action patterns. Requesting does not require acting out the content of a message; directing someone's attention is sufficient ("cooperative request").

One question is then which of the two interpretations is correct for infants' request when they begin pointing around 12 months of age? Differentiating simpler instrumental requests from cooperative communicative requests is important to delineate the ontogenetic and phylogenetic origins of human

(C) 2013 The Authors

Child Development (c) 2013 Society for Research in Child Development, Inc. All rights reserved. 0009-3920/2014/8502-0008

DOI: $10.1111 /$ cdev.12141 
communication. Although apes in captivity have been reported to point imperatively to request food and objects from humans, they do so with the whole hand close to the caging, sometimes banging and vocalizing in concert (Leavens, Hopkins, \& Thomas, 2004). Sometimes they also protrude the index finger through the mesh or hole in the caging, with some indication that the index-finger extension is influenced by the size of the openings in the mesh (Leavens, Ely, Hopkins, \& Bard, 2012). Their gestures are clearly communicative acts and not serious attempts at reaching, as apes do not do this when they are alone (the so-called audience effect; e.g., Call \& Tomasello, 1994; Kaminski, Call, \& Tomasello, 2008). Apes' requests are therefore often interpreted as imperative pointing, akin to human infants' pointing. However, it is not known on which level apes' and infants' requests operate, and whether they reveal phylogenetic similarities or differences in nonverbal gestural communication.

To determine the level of infants' and apes' requests it is important to delineate the situations in which they request. In the prototypical situations, the desirable object is out of reach and inaccessible to the subject. Subjects are thus hindered by environmental constraints to retrieve the objects by themselves. There are subtle differences between the paradigms employed with infants and apes. Infants are usually proximally constrained, for example, by a high chair, and cannot move close enough to the desired object to retrieve it (e.g., Franco \& Butterworth, 1996). A forward-leaning body, as if trying to or signaling the attempt to obtain the object may result from the proximal constraint. On a lean interpretation of infant imperative pointing, if the proximal constraints were removed, then infants should move toward the desired target to signal their retrieval attempt or try to retrieve the item by themselves. In contrast, if infants' requests went beyond signaling retrieval attempts and were based primarily on directing others' attention, then infants should point to the desired items, even when they could approach these by themselves. With regard to apes, the constraints are slightly different. Apes are distally constrained by the caging. They can freely move around within their enclosure and adopt any position, but they cannot access the reward outside the caging. On a lean interpretation of apes' imperative pointing, apes should thus always move as close as possible to the caging that blocks access to the desired item, to make their retrieval attempts apparent to the human keeper. In contrast, if apes' requests went beyond signaling retrieval attempts through their action patterns and was instead primarily based on directing others' attention, apes should sometimes also point from a distance, with hand and arm extended free in the air, instead of approaching the desired items as close as possible.

This study tested the two interpretations of requests in infants and apes, trying to provide evidence against the lean interpretation of imperative pointing. We assessed infants' and apes' performance in comparable situations in which access to desired items was distally blocked by caging. The proximal constraints were removed such that participants could freely move around, and their willingness and motivation to do so was established in a separate locomotion control test. We tested whether infants (Experiment 1) and apes (Experiment 2), when centered at one place, would point from there with hands and arms free in the air, to direct attention to desired items at a distance, instead of approaching the blocked items as close as possible and signal their intention to obtain these through their approach behavior. In Experiment 3, a follow-up on infants' performance in Experiment 1 was done and it was tested whether infants would point to desired items even when they could easily access these by themselves. Recruiting someone's help in tasks that one can solve by oneself reveals a preference to collaborate (Rekers, Haun, \& Tomasello, 2011; Warneken, Gräfenhain, \& Tomasello, 2012) and provides evidence for the interpretation of imperative pointing as a cooperative request.

\section{Experiment 1}

Infants were centered at one side of the room with a game. We tested whether infants would direct the experimenter's attention by pointing from there to desired but blocked items at another place, or whether they would signal their individual attempt of wanting to obtain the objects by moving as close as possible to the blocked items. We manipulated also the distance between pointer and desired item, to determine whether effort to move would increase the likelihood to point. A locomotion control test assured that all infants were motivated and able to crawl to the blocked items.

\section{Method}

\section{Participants}

Twenty 12-month-old infants participated (10 boys; $M=12.8$, range $=12.5-12.9$ ). Infants were 
recruited using a database to which parents voluntarily subscribed to participate in studies. Parents were asked upon recruitment whether their infant already pointed. Only infants who were able to point were invited for testing. One additional infant was tested but excluded because she did not pass the locomotion test (see the Procedure section).

\section{Apparatus}

The study setup consisted of a $190 \times 133 \mathrm{~cm}$ carpet on the floor, with the infant and E1 sitting on one end and E2 with additional objects on the other end (see Figure 1). E1, the infant, and parent each sat on a $0.6 \times 0.6 \mathrm{~m}$ cushion to mark the location for each person. E1 sat to the right of the infant while the parent was situated to the other side of the infant, to the back. E2 sat on a small stool at the other end of the carpet behind a $1.23 \times 0.4 \times$ $0.75 \mathrm{~m}$ wooden cage with mesh in front.

We used two toys for the study. The first was a transparent marble track in which one could insert colored ping-pong balls. The second was a transparent chute in which one could insert colored walnuts. The chute made a xylophone sound each time a walnut was inserted. At the start of a session E1 and the infant always had two objects (balls or walnuts) at their disposal. Eight additional objects were placed on a tray, which was placed behind the mesh in the cage at the other side of the carpet. The infant could see the additional objects. The meshed cage was placed at two different distances from the infant. The far distance was $1.8 \mathrm{~m}$ (distal condition; clearly out of reach) and the close distance $0.95 \mathrm{~m}$ (proximal condition; just out of reach). Distances were blocked and the order of distances was counterbalanced across participants. Infants received two test trials per block, thus totaling four trials.

\section{Procedure}

Before the actual testing session, the two experimenters played with the infant to familiarize her with the surroundings as a warm-up. Each block consisted of a demonstration and a test phase.

Demonstration phase. At the start of the session E1 and the infant had two objects at their disposal. E1 first demonstrated the toy by putting one object into the chute and then encouraged the infant to do the same. When the second object was put in the chute, E1 stated that she wanted to continue ("Yeah, let's do that again!") and subsequently commented on the fact that they were out of objects ("Oh, hmm, all gone!"). E2 responded to this by stating clearly to the infant and E1 that she had more and gave two more objects from the cage to E1, who in turn gave them to the infant to put in the chute. After the infant had put this second pair of objects into the chute the sequence was repeated with a third pair of objects. Infants thus had the opportunity to throw in five objects in three demonstration sequences. The test phase started after the infant had put the third set of objects in the chute.

Test phase. At the end of the third demonstration sequence, E1 stated that she wanted to continue and commented that they were out of objects. This time, however, E2 did not respond but instead was turned to the side, facing away from the play situation and pretending to be occupied with something else. E1 tried to prompt the infant to request for more objects by encouraging her to continue with the game ("This is fun! Let's continue!"). She repeated this sentence twice in case of no immediate response. In case the infant requested by either pointing or crawling to the mesh E1 notified E2 by commenting on the infants' behavior ("Yeah, do you want more?"). E2 would
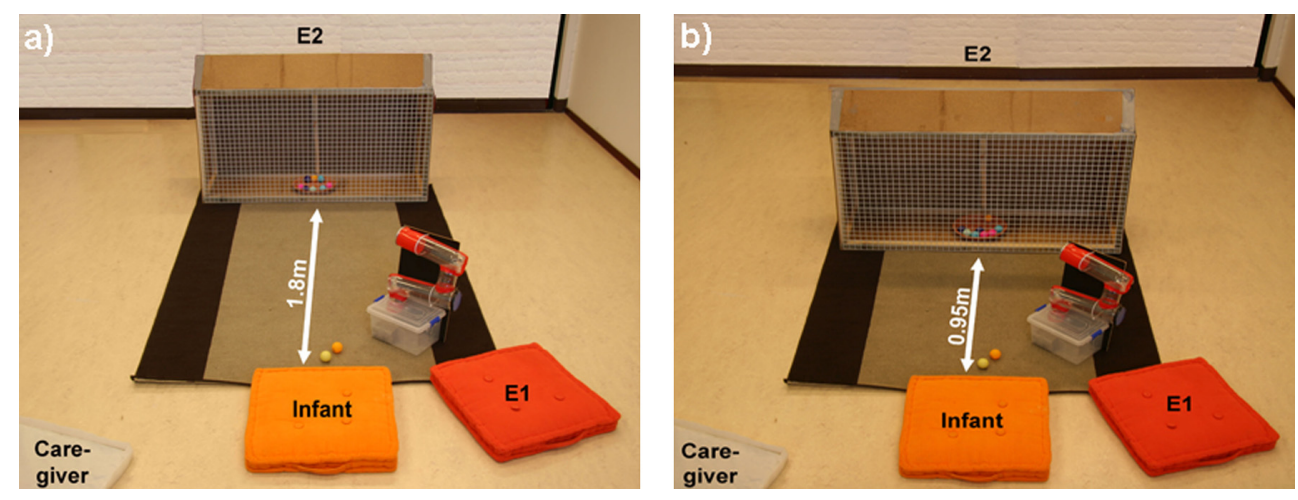

Figure 1. Setup of Experiment 1: The desired items are blocked behind cage mesh (a) at distal distance of $1.8 \mathrm{~m}$ and (b) at proximal distance of $0.95 \mathrm{~m}$. 
then turn back to the play situation and take two objects from the tray to give to E1. E1 then gave them to the infant and they would continue. In case the infant did not respond, E2 delivered more objects to E1 after $20 \mathrm{~s}$. Then the second test trial started with the same procedure. After finishing the first block, E1 and the infant received a new toy from E2, and E2 moved the cage with additional objects to the second distance (proximal or distal; the order was counterbalanced across participants) and the same procedure was repeated for the second block.

Locomotion. We administered a locomotion check in case infants had not moved to the objects during a testing session. At the end of the session, E2 would take a ball and invite the infant to come over and play with the ball from the other side of the room to establish if she was able and motivated to locomote independently to the objects.

\section{Coding and Reliability}

The first response in each test trial was coded and used for analysis, since in theory infants could point multiple times to request during a test trial, but could only crawl over once. A point was coded if the arm was half or fully extended with the index finger protruded ("index-finger point") or the fingers stretched ("hand point"). The hand palm could either be facing up or down. "Crawling" was coded if the infant moved forward on its own to the tray with additional objects. A naive second person coded the four test trials for $30 \%$ of the infants $(N=6)$, yielding a Cohen's $\kappa=.88$ over a percentage agreement of $91 \%$.

\section{Results}

All infants passed the locomotion test and completed more than one test trial. Three infants did not show a response. Nine infants only pointed, and seven infants only crawled. One infant used both strategies (McNemar test, binomial distribution, $p=.804$; see left panel of Figure 2).

Nineteen of the 20 infants completed both trials at both distances ( 1 infant completed only the distal condition). Given the rank data quality (two trials per condition) we conducted nonparametric tests. Figure 3 shows the mean proportion of trials in which infants pointed or crawled as a function of distance. A Wilcoxon signed-rank test revealed no significant difference for the mean proportion of trials in which infants pointed or crawled $(N=20$, $z=1.25, p=.21$ ). However, Wilcoxon tests for each condition revealed that in the distal condition, infants pointed more than they crawled $(N=20$,

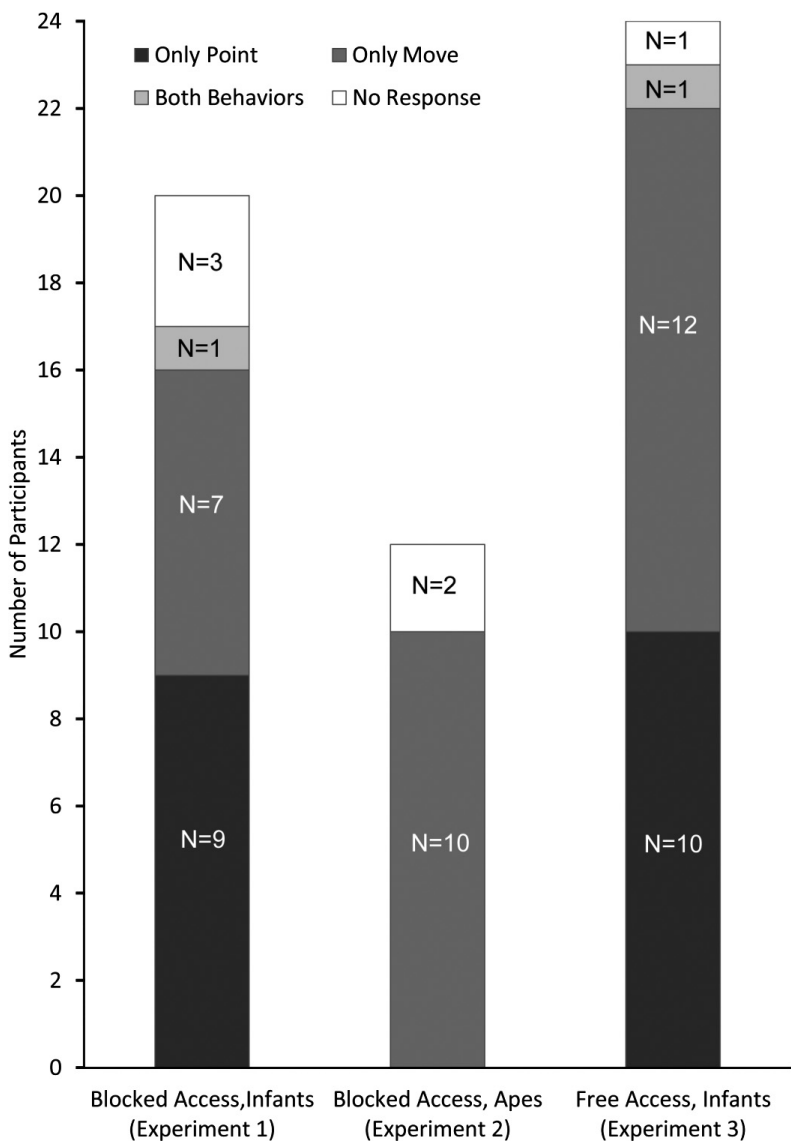

Figure 2. Number of participants who either only pointed, only moved, or displayed both or none of the behaviors to obtain the desired item

$z=2.12, p=.035)$. In the proximal condition, these differences were not significant $(N=19, z=.351$, $p=.726)$. Similar results were obtained with a parametric 2 (behavior) $\times 2$ (distance) $\times 2$ (order) mixed analysis of variance (ANOVA). It yielded a marginal effect of behavior suggesting more pointing than crawling, $F(1,17)=4.28, p=.054$, and a significant interaction between distance and behavior, $F(1,17)=5.64, p=.030$, revealing more pointing than crawling in the distal condition: simple effects, $F(1,17)=11.63, p=.003$. There was also a significant interaction between behavior and order, $F(1$, $17)=7.93, p=.012$, suggesting more pointing than crawling when the distal condition was administered first: simple effects, $F(1,17)=10.31, p=.005$, and a marginal interaction between distance and order, $F(1,17)=4.12, p=.058$, suggesting more behavior in the condition that was administered first (simple effects, $n s$ ).

The majority of points were with the extended index finger $(69 \%)$. In the distal condition, $71 \%$ of the points were with the index finger, and in the 


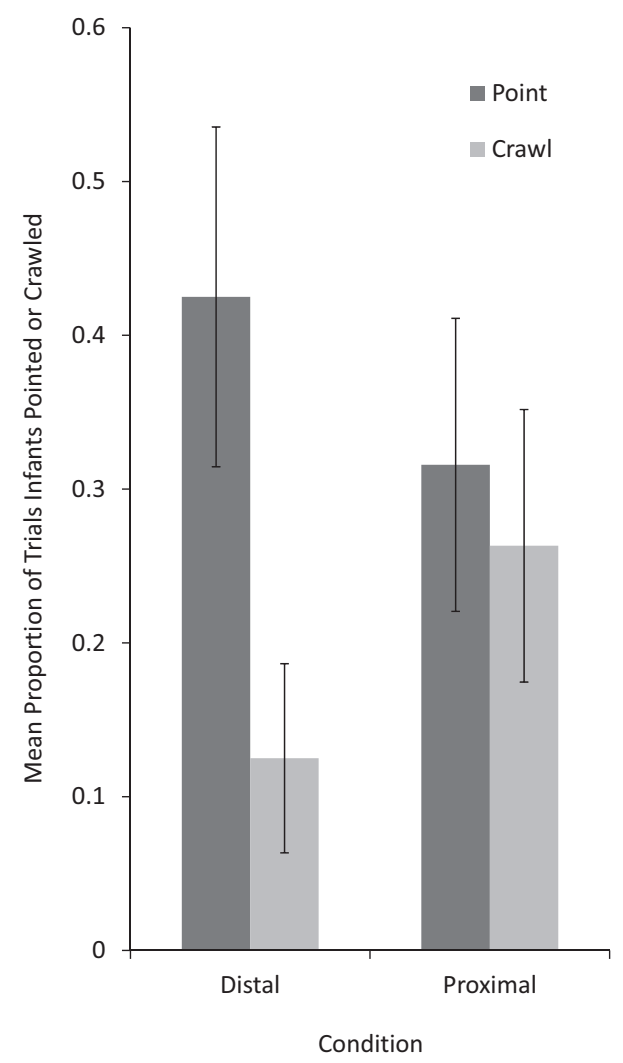

Figure 3. Experiment 1: Mean proportion of trials in which infants pointed or crawled to the blocked items as a function of distance. Error bars represent standard errors of the mean.

proximal condition, $67 \%$ of the points were with the index finger. A within-subject comparison $(N=10)$ on the mean proportion of index-finger points revealed no differences between conditions (Wilcoxon signed-rank test, $z=.322, p=.748$ ).

\section{Discussion}

When proximal constraints were removed and infants could freely move around, infants often remained at their position and pointed from a distance. Infants did not always move as close as possible to the desired objects. This finding thus rejects the lean alternative that infants convey their requests only through their instrumental action patterns of retrieving objects. Instead, findings are consistent with the interpretation that infants' imperative pointing is a cooperative communicative act that directs others' attention within common ground to desired objects, with the expectation that the recipient will do as one wishes.

Infants pointed more often when the objects were farther away, and their points were predominantly done with the index finger. Presumably infants understood that it was more efficient to direct the adult's attention to the distal inaccessible objects than to crawl there. It is important to recall that all infants were able to crawl to the distal objects when prompted to do so in the locomotion test.

About half of the infants pointed and half of them crawled, with only one infant using both strategies. However, while our locomotion check confirmed that all infants could crawl to the objects, infants were not submitted to a similar pointing check in the current setup. It is possible that infants who did not point in the current situation were intimidated or not motivated to point. It is also important to note that infants were not familiarized or exposed to the cage before, so that it is not clear whether all infants knew that they could not open the cage. Indeed, it is possible that infants who did not point simply approached the cage to explore the mesh instead of signaling their request to obtain the blocked items. While it is thus possible that more infants would point under more favorable and controlled situations, the current findings clearly demonstrate that the 12-month-olds who pointed in this setting used a communicative, attention-directing, object-retrieval strategy rather than that they signaled through instrumental action patterns their intentions to obtain an object. Experiment 2 investigated apes' performance in a design analogous to Experiment 1 to test whether apes, too, request like human infants do.

\section{Experiment 2}

Analogous to Experiment 1 we centered apes at one side of the cage by providing them with desirable food items (bananas). Staying at the location was rewarded since food items were offered only at that location. More food items were available at another, distal location. We administered only a distal condition, since the distal condition elicited more pointing in infants and should be more costly in terms of locomotion, thus maximizing the likelihood of eliciting distal pointing. As in Experiment 1 , we tested whether apes would refrain from moving as close as possible to the desired items outside their cage and instead direct a keeper's attention to the items from afar with a freestanding pointing gesture. We reasoned that if apes point imperatively like human 12-month-olds, they should at least sometimes stay put at the location where they always obtained the desired food, and point from there to obtain more food. Alternatively, if apes' imperative pointing is a direct consequence of the 
environmental constraints of the caging, we expected apes to always approach the desirable reward as close as possible or necessary to make their request apparent through their individual action patterns of retrieving objects. We used human keepers instead of conspecifics as recipients since apes have been observed to point only in captivity and only for human keepers, not conspecifics.

\section{Method}

\section{Participants}

We tested 12 chimpanzees (Pan troglodytes) and 4 bonobos (Pan paniscus) at the Wolfgang Köhler Primate Research Center, Leipzig, Germany. All apes were familiar with the zoo setting, testing, and the keepers. Two chimpanzees were hand raised by human keepers in a zoo setting. The rest of the participating individuals had no specific history of enculturation. One chimpanzee and three bonobos were tested in addition, but excluded because of experimenter error (one bonobo) or because they could not be interested in participating in the task. There were five males and seven females with age ranges from 9 to 35 years $(M=17.7)$. Subjects were tested individually, except for two individuals who were accompanied by their infants. All subjects participated voluntarily and were previously or currently participating in other studies so they were used to participating in tests. Also, all subjects had participated in previous pointing studies.

\section{Apparatus}

The participants were tested in a setup similar to that of the infants in Experiment 1. Instead of toys, a food reward in the form of banana pieces was used to ensure that the participants were engaged and motivated to request for more during testing.

Testing occurred in an enclosure that consisted of multiple adjacent mesh cages, which were connected via open sliding doors. E1 sat in the far left (closed off) cage of the enclosure to deliver the food to the participant. E2 was located in front of one of the cages, $6 \mathrm{~m}$ to the right. E1 used a low table to display the food and a transparent food dispenser to deliver the food. E2 had a large transparent bucket filled with banana pieces placed in front of her on a tripod at a height that was easily visible for the participants. The apes had free access to all cages between E1 and E2 (see Figure 4).

The experimenters used a muted walkie-talkie to signal trial start and ending without distracting the participants through additional noise. Pressing the button on one walkie-talkie produced a visible cue on the display of the other walkie-talkie. Three cameras were placed to capture all distance from $\mathrm{E} 1$ to $\mathrm{E} 2$ to be able to record any behavior invisible to E1 and E2 during testing.

\section{Procedure}

The procedure was similar to that of Experiment 1 with some slight adjustments. Every session started with a demonstration phase followed by a test phase. Subjects received four test trials in the distal condition. We used a distance of $6 \mathrm{~m}$, the longest distance possible within the constraints imposed by the enclosure, to increase the incentive to stay centered at the feeding location.

Demonstration phase. The demonstration phase was included to familiarize the apes with the fact that E2 was willing to supply more food in case

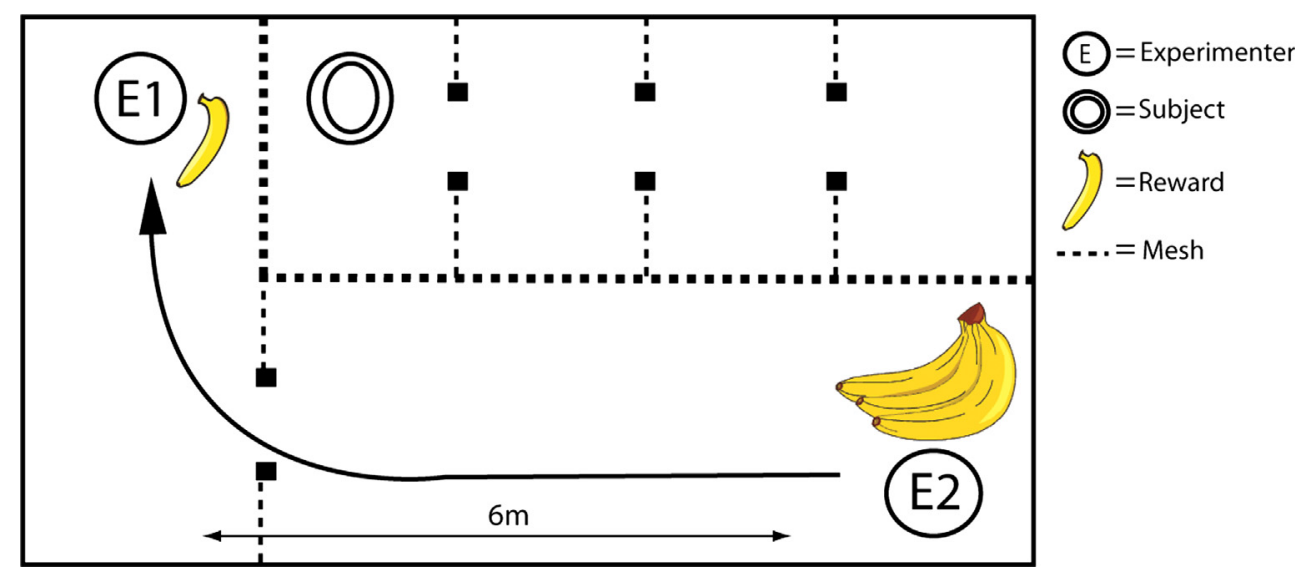

Figure 4. Schematic drawing of the setup in Experiment 2. 
they were out and that E2 would only provide them with more food indirectly, via E1. E1 sat behind the table with four pieces of banana. At the start of the experiment, E1 delivered the four food items piece by piece to the subject with an interval of $8 \mathrm{~s}$. After the participant had finished the last piece of food, E1 signaled E2 via the muted walkietalkie and E2 subsequently walked over to E1 with the bucket of bananas to provide four additional pieces of food to E1, analogous to the infant study. When E2 was back at her position, E1 again delivered the food to the subject. This was repeated three times, making four demonstration sequences in total.

Test phase. After the subject had finished the last piece of food in the demonstration phase, E1 again signaled E2 and the test phase started. Analogous to Experiment 1, E2 did not immediately respond by walking over, but stayed at her position and only supplied more food to E1 upon request of the subject. Participants' behavior was recorded for $1 \mathrm{~min}$. In case the subject requested for more food from E2 from anywhere between E1 and E2, she walked over to E1 to supply more food to E1 and the next test trial started. Only gestural requests were acknowledged with more food; any other behavior (cage banging, vocalizing, etc.) was not counted as a referential request. Each subject received four test trials in total. All test trials were run in the same session as long as the subject kept requesting on each trial. In case subjects did not request during a test trial, the session for that day was ended and continued the next day. Such follow-up sessions again started with a four-trial demonstration phase. Thus, in theory, subjects could receive four sessions on four subsequent days in case they did not respond in any test trial.

\section{Coding and Reliability}

The same main coder of Experiment 1 coded the video recordings of the testing sessions. Points were coded when the arm was fully or half extended, with only the index finger ("index-finger point") or all fingers protruded ("hand point'). In addition, we counted gestures as hand point that involved grabbing the mesh near the reward, if this gesture was repeated several times until E2 responded and walked over to E1. Repetition in this case was regarded as persistence and therefore the gesture was regarded as communicative and intentional. Distal requests were coded if the subject requested from anywhere but the enclosure in front of which E2 was standing, a proximal request involved mov- ing close to the enclosure where the food was located. A naive second coder coded the four test trials for $25 \%$ of the participants $(N=4)$ revealing a Cohen's $K=0.81$ over a percentage agreement of $93 \%$.

\section{Results}

All 12 subjects completed more than one trial. Two of the chimpanzees showed neither of the relevant behaviors on any of the four trials. Of the remaining participants, none pointed from the feeding location to the food items during any of the test trials (see middle panel, Figure 2). Instead, all 10 apes moved as close as possible to the food items behind the cage and requested from there by protruding the hand through the mesh. None of these subjects used index-finger points to request; all of them requested by extending their hands or by hanging their hands in the caging.

Supplementary analyses revealed that all but one subject requested at least once during the demophases to present banana pieces by protruding their finger or hand through the caging (43 index-finger extensions [ 23 by the bonobo alone]; 22 hand points).

\section{Discussion}

The results of Experiment 2 are in line with previous findings (e.g., Leavens, Hopkins, \& Bard, 1996). When great apes request, they move as close as possible to the desired item. They do not direct others' attention from a distance to the desired item when they have the choice of approaching it. This finding supports the original, lean hypothesis that apes signal their requests through their instrumental action patterns of retrieving objects rather than simply directing others' attention within common ground.

Importantly, we found evidence that apes did communicate their requests. Thus, 10 of 12 apes were observed requesting in their prototypical way at least once during the experiment, including index-finger extensions, and previous findings show that these behaviors are not serious action attempts, since the behaviors cease if no one is attending (Call \& Tomasello, 1994; Kaminski et al., 2008; Krause \& Fouts, 1997; Leavens et al., 2004). However, the fact that they always move their body as close as possible toward the desired item suggests that apes are using a strategy of enacting their message rather than presuming the message to be within common ground so that directing attention from afar would suffice. Thus, the distal constraint of the caging, together with the experience of an intention-reading 
cooperative human keeper, presumably lead to captive apes" "pointing" behavior.

Removing the distal constraint would inevitably lead apes to retrieve the desired items by themselves, as is evident from their behavior in their natural habitats. It is not known, however, whether infants, too, would always resort to an individual strategy of retrieving fully accessible objects when given the opportunity or, alternatively, prefer to cooperate and communicate a request even when they could fulfill it by themselves at minimal cost. Slightly older toddlers have been shown to collaborate with a partner in a game they can also play by themselves (Warneken et al., 2012; see also Fawcett \& Liszkowski, 2012). If it could be shown that infants point to request items they could access by themselves, this would provide evidence for a cooperative communicative use of pointing which clearly goes beyond the use of instrumental action patterns. Experiment 3 addressed this issue.

\section{Experiment 3}

In Experiment 3, we modified the paradigm of Experiment 1 by removing not only the proximal but also the distal constraint. We tested whether infants would retrieve items in the open by themselves or, instead, point at them from a distance to communicate their request. As in Experiment 1, we used a proximal and distal distance, to find out whether effort to move would influence the likelihood of communicative requests.

\section{Method}

\section{Participants}

Twenty-four 12-month-old infants participated in Experiment 3 (12 boys; $M=12.8$, range $=12.5-12.9$ ).
Infants again were recruited using a database to which parents voluntarily subscribed to participate in studies. Parents reported that all infants could point and locomote independently. Two additional infants were tested but excluded: one due to fussiness and one infant because it did not pass the locomotion test.

\section{Apparatus and Procedure}

The study used the same setup, procedure, and demonstrations as in Experiment 1, but without the mesh box (see Figure 5). This way, the tray with additional objects was freely accessible to the infants. As in Experiment 1, we presented the infants with two different toys at two different distances, counterbalanced across participants.

\section{Coding and Reliability}

The same main coder of Experiments 1 and 2 coded all video recordings of Experiment 3. We used the same coding scheme as in Experiment 1. A naive second coder coded test trials of $20 \%$ of the participants $(N=5)$ yielding a Cohen's $\kappa$ of 1 (100\% agreement).

\section{Results}

All infants passed the locomotion test and completed at least one trial. One infant did not show a response. Ten infants only pointed and 12 infants only crawled. One infant used both strategies (McNemar test, binomial distribution, $p=.832$; see right panel of Figure 2).

Sixteen of the 24 infants completed at least one trial in both distances (of these, 14 infants completed both trials at both distances). Figure 6 shows the mean proportion of trials in which infants pointed or crawled as a function of distance. A Wilcoxon
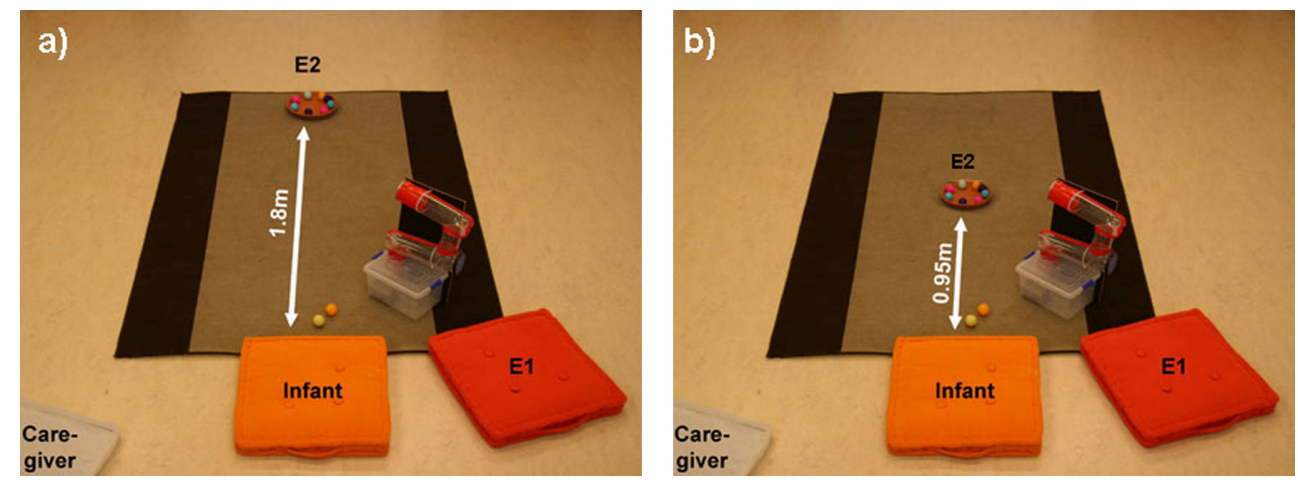

Figure 5. Setup of Experiment 3: The objects are fully accessible (a) at a distal distance of $1.8 \mathrm{~m}$ and (b) at a proximal distance of $0.95 \mathrm{~m}$. 


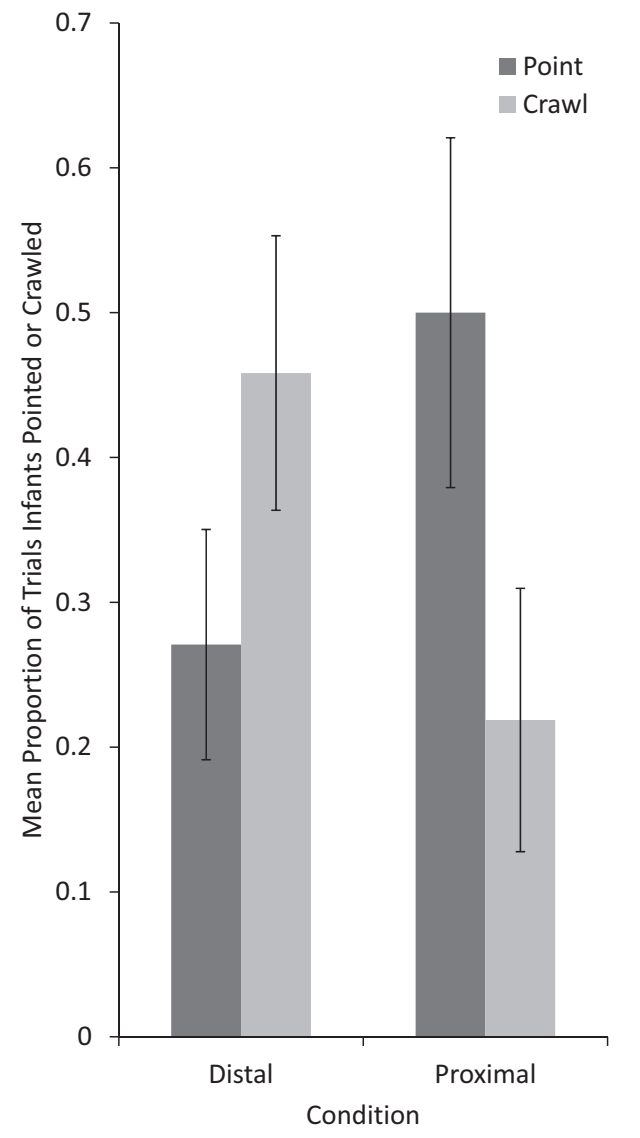

Figure 6. Experiment 3: Mean proportion of trials in which infants pointed or crawled to the fully accessible items as a function of distance. Error bars represent standard errors of the mean.

signed-rank test showed no significant difference for the mean proportion of trials in which infants pointed or crawled $(N=24, z=.361, p=.718)$. Wilcoxon signed-rank tests for each condition revealed that infants did not point or crawl more when the object was far $(N=24, z=1.25, p=.208)$ or close $(N=16, z=1.52, p=.126)$. Similar results were obtained with a parametric 2 (behavior) $\times 2$ (distance) $\times 2$ (order) ANOVA. It yielded no main effects and a significant interaction between behavior and distance, $F(1,14)=7.59, p=.015$. Simple effects revealed no significant differences between behaviors for either distance and suggested a tendency for more pointing in the proximal than the distal condition, $F(1,14)=4.43, p=.054$.

As for the hand shape, the mean percentage of index-finger points was $58 \%$. In the distal condition, $58 \%$ of the points were with the index finger, and in the proximal condition $71 \%$ of the points were with the index finger. A within-subject comparison $(N=11)$ on the mean proportion of index-finger points revealed a trend for more index-finger points in the proximal condition than the distal condition (Wilcoxon signed-rank test, $z=1.89, p=.059$ ).

\section{Discussion}

When all constraints were removed such that infants could freely approach the desired objects and retrieve these by themselves, many infants still pointed to communicate their request. Often, they pointed with an extended index finger as adults do when directing attention to request specific entities within common ground. The distance from infant to the desired object did not influence infants' pointing. Even when the objects were fairly close and obtainable at minimal cost, infants still chose to communicate a request by pointing.

As in Experiment 1, there were individual differences in strategies of object retrieval. Only one of the infants used both communicative and individual strategies, while the rest chose either one consistently. Importantly, however, all infants were able to retrieve the objects by themselves when invited to do so after the test session. As in Experiment 1, the procedure did not involve a pointing check, and so it remains possible that some infants were inhibited or unmotivated to point in the current lab situation. However, those infants who did point could also obtain the objects by themselves. The stability of these individual differences was not addressed in this study. However, there is developing increase in reliance on cooperative strategies in children's verbal request (see Shwe \& Markman, 1997).

The results show that 12-month-old infants can choose a communicative over an instrumental strategy. These findings are in line with previous findings, which show that toddlers cooperate even when they can achieve an instrumental goal by themselves (Warneken et al., 2012). Choosing to recruit a partner instead of relying on oneself reveals infants' social orientation and supports the hypothesis that by 12 months, preverbal infants' imperative pointing already is a cooperative communicative act.

\section{General Discussion}

A "lean" interpretation of nonverbal gestural requests holds that infants and apes signal their intention to obtain an item with action patterns that are instrumental in achieving the goal. On this interpretation subjects make their object-directed intention apparent, for example, by approaching 
the desired object as close as possible or necessary to signal their intention. If the desired object is accessible, the goal of obtaining it is achieved individually by executing the instrumental action, since there is no need to communicate one's desire. In essence, in this view communication arises from environmental constraints that prevent subjects from achieving a goal by themselves (Vauclair, 1990). However, human requests, like other communicative acts, build on cooperative principles that emerged some time in phylogeny and must develop in ontogeny. When an adult points to a salt dispenser on the dinner table, it is barely the consequence of a principal impossibility to obtain the dispenser, certainly not when it is close by. Instead, human communicative acts build on cooperative expectations of acting together and on an understanding of each other's communicative actions within a shared context (Clark, 1996; Tomasello, 2008). In this alternative view, requests do not depend on environmental constraints. Instead, these requests depend on cooperative expectations within shared common ground, which make it possible to simply direct others' attention, without the necessity to act out the content of the message. Such decontextualized requests, like canonical index finger pointing, are not based on instrumental action patterns (see Bruner, 1975).

This study demonstrates that when 12-month-old infants point to request they communicate on a cooperative level, independent of environmental constraints, and not through instrumental action patterns, like reaching. The study also demonstrates that nonhuman primates do not request in this way. Instead, they make their intention to obtain an item apparent through the instrumental action patterns of obtaining it. Main findings were that when removing all constraints, infants often directed attention from a distance to request objects from others communicatively. This was even the case when the objects were close by and fully accessible. Unlike infants, apes never directed attention to the desired items from afar. Instead, they signaled their requests by approaching the desired items as close as possible. Rather than directing attention within common ground, apes made their intention to obtain the items apparent through their overt approach behavior. While the finding with apes support the idea that object requests in nonhuman primates arise as a direct consequence of environmental constraints (e.g., Vauclair, 1990), the findings with infants show that around 12 months of age infants' requests turn into human cooperative communication, reflecting a preference for collabo- ration rather than attempts to overcome constraints in instrumental acting.

The difference between infants' and apes' requests presumably stems from apes' lack of the primary communicative goal to direct others' attention to desired items outside the dyad. Apes' natural gestural communication is an outcome of interpreting and abbreviating individual actions ("ontogenetic ritualization"; Tomasello, 2008), with little evidence that apes arbitrarily direct attention to things outside the dyad (apes' food-begging gesture is usually directed at something currently in possession of the addressee and reflects tolerated attempts of obtaining food from feeding conspecifics rather than directing attention to it). One possibility is that apes do not coconstruct a cooperative common ground within which directing attention would become meaningful as an object-directed request. For example, studies show that apes, unlike infants, do not use a hiding context to infer that a point to a bucket refers to the hidden item under the bucket (Hare \& Tomasello, 2004). And unlike infants, apes do not use shared experience to request absent items by directing attention to their habitual empty places (Liszkowski, Schäfer, Carpenter, \& Tomasello, 2009). It is doubtful that home-rearing or other enculturation practices would yield substantial changes in apes' cooperative competencies. A study with hand raised juvenile chimpanzees confirmed a selective deficit in tasks requiring a shared common ground or collaborative motivation (Tomasello \& Carpenter, 2005). Furthermore, a reanalysis of sign-language-trained chimpanzees' requests revealed that they used these signs as "wild cards," typically accompanied by instrumental behaviors, to get anything from human keepers (Rivas, 2005). Interestingly, apes can control their actions to conceal their behavior in competitive contexts (Hare, Call, \& Tomasello, 2006). Conversely, cooperative settings with human keepers require control of action in the sense of intentionally revealing the behavioral attempt. Some evidence suggests that apes' instrumental attempts of obtaining items indeed does involve exaggerated action patterns, as sometimes seen in accompanying fretting and banging behaviors (Leavens et al., 2004).

In contrast to apes, human infants naturally point to direct attention to external entities in ample situations, for example, to inform others or to share interest with them. Such pointing works because it is meaningful in the background of a shared understanding of the interactional situation in which it is used (Tomasello, Carpenter, \& Liszkowski, 2007). 
Current findings show that when infants point imperatively they are directing others' attention within common ground, just like when they point declaratively, with the expectation that directing others' attention will spur others into action and make them do as one wishes. Notably, the form of imperative pointing often entails the otherwise nonfunctional extended index finger, as in declarative acts. By 12 months of age, many infants have thus developed some expectation that their attentiondirecting gesture can function as a request in the appropriate circumstances and will be complied with by a cooperative adult. The finding that some infants even communicate a request when they can effortlessly retrieve the object by themselves reveals a preference to collaborate in infants even younger than previously shown (Rekers et al., 2011; Warneken et al., 2012). Infants' social interactional experiences of communicating and joint acting in the 1st year of life presumably transform ontogenetically earlier individual attempts, like reaching for objects, into cooperative communicative acts, where the motivation to cooperate becomes as important as the motivation to reach an individual goal (see Grosse, Behne, Carpenter, \& Tomasello, 2010; Shwe \& Markman, 1997). It is entirely possible that social experience shapes development from early on, as cultural variation in infants' interactional experience suggests (Salomo \& Liszkowski, 2012). Infants' developing skills of directing others' attention to relevant entities and events within shared interactional situations, together with their developing expectations for joint acting and an understanding of actions then give rise to the uniquely human cooperative communicative act of quotidian imperative pointing, already before language has emerged in earnest.

\section{References}

Baron-Cohen, S. (1989). Perceptual role taking and protodeclarative pointing in autism. British Journal of Developmental Psychology, 7, 113-127. doi:10.1111/j.2044-835X.1989.tb00 793.x

Blake, J., O'Rourke, P., \& Borzellino, G. (1994). Form and function in the development of pointing and reaching gestures. Infant Behavior and Development, 17, 195-203. doi:10.1016/0163-6383(94)90055-8

Bruner, J. S. (1975). From communication to language-A psychological perspective. Cognition, 3, 255-287. doi:10. 1016/0010-0277(74)90012-2

Call, J., \& Tomasello, M. (1994). Production and comprehension of referential pointing by orangutans (Pongo pygmaeus). Journal of Comparative Psychology, 108, 307317.
Camaioni, L. (1993). The development of intentional communication: A re-analysis. In J. Nadel \& L. Camaioni (Eds.), New perspectives in early communicative development (pp. 82-96). London: Routledge.

Camaioni, L., Perucchini, P., Bellagamba, F., \& Colonnesi, C. (2004). The role of declarative pointing in developing a theory of mind. Infancy, 5, 291-308. doi:10.1207/ s15327078in0503_3

Carpenter, M., Nagell, K., \& Tomasello, M. (1998). Social cognition, joint attention, and communicative competence from 9 to 15 months of age. Monographs of the Society for Research in Child Development, 63(Serial No. 255).

Clark, H. H. (1996). Using language. Cambridge, UK: Cambridge University Press.

Fawcett, C., \& Liszkowski, U. (2012). Mimicry and play initiation in 18-month old infants. Infant Behavior and Development, 35, 689-696. doi:10.1016/j.infbeh.2012.07. 014

Franco, F., \& Butterworth, G. (1996). Pointing and social awareness: Declaring and requesting in the second year. Journal of Child Language, 23, 307-336. doi:10. 1017/S0305000900008813

Gómez, J. C., Sarriá, E., \& Tamarit, J. (1993). The comparative study of early communication and theories of mind: Ontogeny, phylogeny, and pathology. In S. Baron-Cohen, H. Tager-Flusberg, \& D. J. Cohen (Eds.), Understanding other minds: Perspectives from autism (pp. 397-426). New York: Oxford University Press.

Grosse, G., Behne, T., Carpenter, M., \& Tomasello, M. (2010). Infants communicate in order to be understood. Developmental Psychology, 46, 1710-1722. doi:10.1037/a0020727

Hare, B., Call, J., \& Tomasello, M. (2006). Chimpanzees deceive a human competitor by hiding. Cognition, 101, 495-514. doi:10.1016/j.cognition.2005.01.011

Hare, B., \& Tomasello, M. (2004). Chimpanzees are more skilful at competitive than cooperative cognitive tasks. Animal Behavior, 68, 571-581. doi:10.1016/j.anbehav. 2003.11.011

Kaminski, J., Call, J., \& Tomasello, M. (2008). Chimpanzees know what others know, but not what they believe. Cognition, 109, 224-234. doi:10.1016/j.cognition. 2008.08.010

Krause, M. A., \& Fouts, R. S. (1997). Chimpanzee (Pan troglodytes) pointing: Hand shapes, accuracy, and the role of eye gaze. Journal of Comparative Psychology, 111, 330-336. doi:10.1037/0735-7036.111.4.330

Leavens, D. A., Ely, J., Hopkins, W. D., \& Bard, K. A. (2012). Effects of cage mesh on pointing: Hand shapes in chimpanzees (Pan troglodytes). Animal Cognition, 15, 437-441. doi:10.1007/s10071-011-0466-6

Leavens, D. A., \& Hopkins, W. D. (1999). The wholehand point: The structure and function of pointing from a comparative perspective. Journal of Comparative Psychology, 113, 417-425. doi:10.1037/0735-7036.113.4. 417

Leavens, D. A., Hopkins, W. D., \& Bard, K. A. (1996). Indexical and referential pointing in chimpanzees (Pan 
troglodytes). Journal of Comparative Psychology, 110, 346-353. doi:10.1037/0735-7036.110.4.346

Leavens, D. A., Hopkins, W. D., \& Thomas, R. K. (2004). Referential communication by chimpanzees (Pan troglodytes). Journal of Comparative Psychology, 118, 48-57. doi:10.1037/0735-7036.118.1.48

Liszkowski, U., Schäfer, M., Carpenter, M., \& Tomasello, M. (2009). Pre-linguistic infants, but not chimpanzees, communicate about absent entities. Psychological Science, 20, 654-660. doi:10.1111/j.1467-9280.2009.02346.x

Rekers, Y., Haun, D. B. M., \& Tomasello, M. (2011). Children, but not chimpanzees, prefer to collaborate. Current Biology, 21, 1756-1758. doi:10.1016/j.cub.2011. 08.066

Rivas, E. (2005). Recent use of signs by chimpanzees (Pan troglodytes) in interactions with humans. Journal of Comparative Psychology, 119, 404-417. doi:10.1037/ 0735-7036.119.4.404

Salomo, D., \& Liszkowski, U. (2012). Sociocultural settings influence the emergence of prelinguistic deictic gestures. Child Development. doi:10.1111/cdev.12026

Shwe, H. I., \& Markman, E. M. (1997). Young children's appreciation of the mental impact of their communica- tive signals. Developmental Psychology, 33, 630-636. doi:10.1037/0012-1649.33.4.630

Tomasello, M. (2008). Origins of human communication. Cambridge, MA: MIT Press.

Tomasello, M., \& Camaioni, L. (1997). A comparison of the gestural communication of apes and human infants. Human Development, 40, 7-24. doi:10.1159/000278540

Tomasello, M., \& Carpenter, M. (2005). The emergence of social cognition in three young chimpanzees. Monographs of the Society for Research in Child Development, 70(1, Serial No. 279). doi:10.1111/j.1540-5834.2005.00324.x

Tomasello, M., Carpenter, M., \& Liszkowski, U. (2007). A new look at infant pointing. Child Development, 78, 705-722. doi:10.1111/j.1467-8624.2007.01025.x

Vauclair, J. (1990). Primate cognition: From representation to language. In S. T. Parker \& K. R. Gibson (Eds.), "Language" and intellect in monkeys and apes (pp. 312329). Cambridge, UK: Cambridge University Press.

Warneken, F., Gräfenhain, M., \& Tomasello, M. (2012). Collaborative partner or social tool? New evidence for young children's understanding of joint intentions in collaborative activities. Developmental Science, 15, 54-61. doi:10.1111/j.1467-7687.2011.01107.x 